\title{
Admissible Rules and the Leibniz Hierarchy
}

\author{
James G. Raftery
}

\begin{abstract}
This paper provides a semantic analysis of admissible rules and associated completeness conditions for arbitrary deductive systems, using the framework of abstract algebraic logic. Algebraizability is not assumed, so the meaning and significance of the principal notions vary with the level of the Leibniz hierarchy at which they are presented. As a case study of the resulting theory, the nonalgebraizable fragments of relevance logic are considered.
\end{abstract}

\section{Introduction}

Many researchers have considered the question: to what extent can we interpret a logic plausibly in its own metalanguage? Disjunction properties are one manifestation of this concern. A problem in the reverse spirit is the derivability of admissible rules. Following Lorenzen [29], we say that a rule of inference is admissible in a formal system if its addition to the system produces no new theorems. A simple example is the rule of necessitation, $x / \square x$, which is admissible (and not derivable) in quasinormal modal logics. Less trivially, the process of cut-elimination shows that underivable cut rules are admissible in suitable sequent calculi.

The algebraizable logics of Blok and Pigozzi [7] constitute the framework for some prominent treatments of admissibility, such as Rybakov's monograph [62]. On the other hand, the quasinormal modal systems and the cut-free subsystems of substructural logics are not algebraizable. The present paper analyzes the semantics of admissible rules in the context of arbitrary deductive systems, indicating which tools of abstract algebraic logic (see Czelakowski [15] and Font, Jansana, and Pigozzi [21]) are really needed at various stages of the theory, while also supplying some new results. The paper is largely self-contained, but its purpose is not to survey the

Received May 1, 2012; accepted June 20, 2013

First published online September 1, 2016

2010 Mathematics Subject Classification: Primary 03B22, 03G27; Secondary 03B47, 08C10

Keywords: deductive system, admissible rule, reduced matrix, structural completeness, Leibniz hierarchy, [order] algebraizable logic, BCIW

(C) 2016 by University of Notre Dame 10.1215/00294527-3671151 


\title{
Admissible Rules and the Leibniz Hierarchy
}

\author{
James G. Raftery
}

\begin{abstract}
This paper provides a semantic analysis of admissible rules and associated completeness conditions for arbitrary deductive systems, using the framework of abstract algebraic logic. Algebraizability is not assumed, so the meaning and significance of the principal notions vary with the level of the Leibniz hierarchy at which they are presented. As a case study of the resulting theory, the nonalgebraizable fragments of relevance logic are considered.
\end{abstract}

\section{Introduction}

Many researchers have considered the question: to what extent can we interpret a logic plausibly in its own metalanguage? Disjunction properties are one manifestation of this concern. A problem in the reverse spirit is the derivability of admissible rules. Following Lorenzen [29], we say that a rule of inference is admissible in a formal system if its addition to the system produces no new theorems. A simple example is the rule of necessitation, $x / \square x$, which is admissible (and not derivable) in quasinormal modal logics. Less trivially, the process of cut-elimination shows that underivable cut rules are admissible in suitable sequent calculi.

The algebraizable logics of Blok and Pigozzi [7] constitute the framework for some prominent treatments of admissibility, such as Rybakov's monograph [62]. On the other hand, the quasinormal modal systems and the cut-free subsystems of substructural logics are not algebraizable. The present paper analyzes the semantics of admissible rules in the context of arbitrary deductive systems, indicating which tools of abstract algebraic logic (see Czelakowski [15] and Font, Jansana, and Pigozzi [21]) are really needed at various stages of the theory, while also supplying some new results. The paper is largely self-contained, but its purpose is not to survey the

Received May 1, 2012; accepted June 20, 2013

First published online September 1, 2016

2010 Mathematics Subject Classification: Primary 03B22, 03G27; Secondary 03B47, 08C10

Keywords: deductive system, admissible rule, reduced matrix, structural completeness, Leibniz hierarchy, [order] algebraizable logic, BCIW

(C) 2016 by University of Notre Dame 10.1215/00294527-3671151 


\title{
Admissible Rules and the Leibniz Hierarchy
}

\author{
James G. Raftery
}

\begin{abstract}
This paper provides a semantic analysis of admissible rules and associated completeness conditions for arbitrary deductive systems, using the framework of abstract algebraic logic. Algebraizability is not assumed, so the meaning and significance of the principal notions vary with the level of the Leibniz hierarchy at which they are presented. As a case study of the resulting theory, the nonalgebraizable fragments of relevance logic are considered.
\end{abstract}

\section{Introduction}

Many researchers have considered the question: to what extent can we interpret a logic plausibly in its own metalanguage? Disjunction properties are one manifestation of this concern. A problem in the reverse spirit is the derivability of admissible rules. Following Lorenzen [29], we say that a rule of inference is admissible in a formal system if its addition to the system produces no new theorems. A simple example is the rule of necessitation, $x / \square x$, which is admissible (and not derivable) in quasinormal modal logics. Less trivially, the process of cut-elimination shows that underivable cut rules are admissible in suitable sequent calculi.

The algebraizable logics of Blok and Pigozzi [7] constitute the framework for some prominent treatments of admissibility, such as Rybakov's monograph [62]. On the other hand, the quasinormal modal systems and the cut-free subsystems of substructural logics are not algebraizable. The present paper analyzes the semantics of admissible rules in the context of arbitrary deductive systems, indicating which tools of abstract algebraic logic (see Czelakowski [15] and Font, Jansana, and Pigozzi [21]) are really needed at various stages of the theory, while also supplying some new results. The paper is largely self-contained, but its purpose is not to survey the

Received May 1, 2012; accepted June 20, 2013

First published online September 1, 2016

2010 Mathematics Subject Classification: Primary 03B22, 03G27; Secondary 03B47, 08C10

Keywords: deductive system, admissible rule, reduced matrix, structural completeness, Leibniz hierarchy, [order] algebraizable logic, BCIW

(C) 2016 by University of Notre Dame 10.1215/00294527-3671151 


\title{
Admissible Rules and the Leibniz Hierarchy
}

\author{
James G. Raftery
}

\begin{abstract}
This paper provides a semantic analysis of admissible rules and associated completeness conditions for arbitrary deductive systems, using the framework of abstract algebraic logic. Algebraizability is not assumed, so the meaning and significance of the principal notions vary with the level of the Leibniz hierarchy at which they are presented. As a case study of the resulting theory, the nonalgebraizable fragments of relevance logic are considered.
\end{abstract}

\section{Introduction}

Many researchers have considered the question: to what extent can we interpret a logic plausibly in its own metalanguage? Disjunction properties are one manifestation of this concern. A problem in the reverse spirit is the derivability of admissible rules. Following Lorenzen [29], we say that a rule of inference is admissible in a formal system if its addition to the system produces no new theorems. A simple example is the rule of necessitation, $x / \square x$, which is admissible (and not derivable) in quasinormal modal logics. Less trivially, the process of cut-elimination shows that underivable cut rules are admissible in suitable sequent calculi.

The algebraizable logics of Blok and Pigozzi [7] constitute the framework for some prominent treatments of admissibility, such as Rybakov's monograph [62]. On the other hand, the quasinormal modal systems and the cut-free subsystems of substructural logics are not algebraizable. The present paper analyzes the semantics of admissible rules in the context of arbitrary deductive systems, indicating which tools of abstract algebraic logic (see Czelakowski [15] and Font, Jansana, and Pigozzi [21]) are really needed at various stages of the theory, while also supplying some new results. The paper is largely self-contained, but its purpose is not to survey the

Received May 1, 2012; accepted June 20, 2013

First published online September 1, 2016

2010 Mathematics Subject Classification: Primary 03B22, 03G27; Secondary 03B47, 08C10

Keywords: deductive system, admissible rule, reduced matrix, structural completeness, Leibniz hierarchy, [order] algebraizable logic, BCIW

(C) 2016 by University of Notre Dame 10.1215/00294527-3671151 


\title{
Admissible Rules and the Leibniz Hierarchy
}

\author{
James G. Raftery
}

\begin{abstract}
This paper provides a semantic analysis of admissible rules and associated completeness conditions for arbitrary deductive systems, using the framework of abstract algebraic logic. Algebraizability is not assumed, so the meaning and significance of the principal notions vary with the level of the Leibniz hierarchy at which they are presented. As a case study of the resulting theory, the nonalgebraizable fragments of relevance logic are considered.
\end{abstract}

\section{Introduction}

Many researchers have considered the question: to what extent can we interpret a logic plausibly in its own metalanguage? Disjunction properties are one manifestation of this concern. A problem in the reverse spirit is the derivability of admissible rules. Following Lorenzen [29], we say that a rule of inference is admissible in a formal system if its addition to the system produces no new theorems. A simple example is the rule of necessitation, $x / \square x$, which is admissible (and not derivable) in quasinormal modal logics. Less trivially, the process of cut-elimination shows that underivable cut rules are admissible in suitable sequent calculi.

The algebraizable logics of Blok and Pigozzi [7] constitute the framework for some prominent treatments of admissibility, such as Rybakov's monograph [62]. On the other hand, the quasinormal modal systems and the cut-free subsystems of substructural logics are not algebraizable. The present paper analyzes the semantics of admissible rules in the context of arbitrary deductive systems, indicating which tools of abstract algebraic logic (see Czelakowski [15] and Font, Jansana, and Pigozzi [21]) are really needed at various stages of the theory, while also supplying some new results. The paper is largely self-contained, but its purpose is not to survey the

Received May 1, 2012; accepted June 20, 2013

First published online September 1, 2016

2010 Mathematics Subject Classification: Primary 03B22, 03G27; Secondary 03B47, 08C10

Keywords: deductive system, admissible rule, reduced matrix, structural completeness, Leibniz hierarchy, [order] algebraizable logic, BCIW

(C) 2016 by University of Notre Dame 10.1215/00294527-3671151 


\title{
Admissible Rules and the Leibniz Hierarchy
}

\author{
James G. Raftery
}

\begin{abstract}
This paper provides a semantic analysis of admissible rules and associated completeness conditions for arbitrary deductive systems, using the framework of abstract algebraic logic. Algebraizability is not assumed, so the meaning and significance of the principal notions vary with the level of the Leibniz hierarchy at which they are presented. As a case study of the resulting theory, the nonalgebraizable fragments of relevance logic are considered.
\end{abstract}

\section{Introduction}

Many researchers have considered the question: to what extent can we interpret a logic plausibly in its own metalanguage? Disjunction properties are one manifestation of this concern. A problem in the reverse spirit is the derivability of admissible rules. Following Lorenzen [29], we say that a rule of inference is admissible in a formal system if its addition to the system produces no new theorems. A simple example is the rule of necessitation, $x / \square x$, which is admissible (and not derivable) in quasinormal modal logics. Less trivially, the process of cut-elimination shows that underivable cut rules are admissible in suitable sequent calculi.

The algebraizable logics of Blok and Pigozzi [7] constitute the framework for some prominent treatments of admissibility, such as Rybakov's monograph [62]. On the other hand, the quasinormal modal systems and the cut-free subsystems of substructural logics are not algebraizable. The present paper analyzes the semantics of admissible rules in the context of arbitrary deductive systems, indicating which tools of abstract algebraic logic (see Czelakowski [15] and Font, Jansana, and Pigozzi [21]) are really needed at various stages of the theory, while also supplying some new results. The paper is largely self-contained, but its purpose is not to survey the

Received May 1, 2012; accepted June 20, 2013

First published online September 1, 2016

2010 Mathematics Subject Classification: Primary 03B22, 03G27; Secondary 03B47, 08C10

Keywords: deductive system, admissible rule, reduced matrix, structural completeness, Leibniz hierarchy, [order] algebraizable logic, BCIW

(C) 2016 by University of Notre Dame 10.1215/00294527-3671151 


\title{
Admissible Rules and the Leibniz Hierarchy
}

\author{
James G. Raftery
}

\begin{abstract}
This paper provides a semantic analysis of admissible rules and associated completeness conditions for arbitrary deductive systems, using the framework of abstract algebraic logic. Algebraizability is not assumed, so the meaning and significance of the principal notions vary with the level of the Leibniz hierarchy at which they are presented. As a case study of the resulting theory, the nonalgebraizable fragments of relevance logic are considered.
\end{abstract}

\section{Introduction}

Many researchers have considered the question: to what extent can we interpret a logic plausibly in its own metalanguage? Disjunction properties are one manifestation of this concern. A problem in the reverse spirit is the derivability of admissible rules. Following Lorenzen [29], we say that a rule of inference is admissible in a formal system if its addition to the system produces no new theorems. A simple example is the rule of necessitation, $x / \square x$, which is admissible (and not derivable) in quasinormal modal logics. Less trivially, the process of cut-elimination shows that underivable cut rules are admissible in suitable sequent calculi.

The algebraizable logics of Blok and Pigozzi [7] constitute the framework for some prominent treatments of admissibility, such as Rybakov's monograph [62]. On the other hand, the quasinormal modal systems and the cut-free subsystems of substructural logics are not algebraizable. The present paper analyzes the semantics of admissible rules in the context of arbitrary deductive systems, indicating which tools of abstract algebraic logic (see Czelakowski [15] and Font, Jansana, and Pigozzi [21]) are really needed at various stages of the theory, while also supplying some new results. The paper is largely self-contained, but its purpose is not to survey the

Received May 1, 2012; accepted June 20, 2013

First published online September 1, 2016

2010 Mathematics Subject Classification: Primary 03B22, 03G27; Secondary 03B47, 08C10

Keywords: deductive system, admissible rule, reduced matrix, structural completeness, Leibniz hierarchy, [order] algebraizable logic, BCIW

(C) 2016 by University of Notre Dame 10.1215/00294527-3671151 


\title{
Admissible Rules and the Leibniz Hierarchy
}

\author{
James G. Raftery
}

\begin{abstract}
This paper provides a semantic analysis of admissible rules and associated completeness conditions for arbitrary deductive systems, using the framework of abstract algebraic logic. Algebraizability is not assumed, so the meaning and significance of the principal notions vary with the level of the Leibniz hierarchy at which they are presented. As a case study of the resulting theory, the nonalgebraizable fragments of relevance logic are considered.
\end{abstract}

\section{Introduction}

Many researchers have considered the question: to what extent can we interpret a logic plausibly in its own metalanguage? Disjunction properties are one manifestation of this concern. A problem in the reverse spirit is the derivability of admissible rules. Following Lorenzen [29], we say that a rule of inference is admissible in a formal system if its addition to the system produces no new theorems. A simple example is the rule of necessitation, $x / \square x$, which is admissible (and not derivable) in quasinormal modal logics. Less trivially, the process of cut-elimination shows that underivable cut rules are admissible in suitable sequent calculi.

The algebraizable logics of Blok and Pigozzi [7] constitute the framework for some prominent treatments of admissibility, such as Rybakov's monograph [62]. On the other hand, the quasinormal modal systems and the cut-free subsystems of substructural logics are not algebraizable. The present paper analyzes the semantics of admissible rules in the context of arbitrary deductive systems, indicating which tools of abstract algebraic logic (see Czelakowski [15] and Font, Jansana, and Pigozzi [21]) are really needed at various stages of the theory, while also supplying some new results. The paper is largely self-contained, but its purpose is not to survey the

Received May 1, 2012; accepted June 20, 2013

First published online September 1, 2016

2010 Mathematics Subject Classification: Primary 03B22, 03G27; Secondary 03B47, 08C10

Keywords: deductive system, admissible rule, reduced matrix, structural completeness, Leibniz hierarchy, [order] algebraizable logic, BCIW

(C) 2016 by University of Notre Dame 10.1215/00294527-3671151 


\title{
Admissible Rules and the Leibniz Hierarchy
}

\author{
James G. Raftery
}

\begin{abstract}
This paper provides a semantic analysis of admissible rules and associated completeness conditions for arbitrary deductive systems, using the framework of abstract algebraic logic. Algebraizability is not assumed, so the meaning and significance of the principal notions vary with the level of the Leibniz hierarchy at which they are presented. As a case study of the resulting theory, the nonalgebraizable fragments of relevance logic are considered.
\end{abstract}

\section{Introduction}

Many researchers have considered the question: to what extent can we interpret a logic plausibly in its own metalanguage? Disjunction properties are one manifestation of this concern. A problem in the reverse spirit is the derivability of admissible rules. Following Lorenzen [29], we say that a rule of inference is admissible in a formal system if its addition to the system produces no new theorems. A simple example is the rule of necessitation, $x / \square x$, which is admissible (and not derivable) in quasinormal modal logics. Less trivially, the process of cut-elimination shows that underivable cut rules are admissible in suitable sequent calculi.

The algebraizable logics of Blok and Pigozzi [7] constitute the framework for some prominent treatments of admissibility, such as Rybakov's monograph [62]. On the other hand, the quasinormal modal systems and the cut-free subsystems of substructural logics are not algebraizable. The present paper analyzes the semantics of admissible rules in the context of arbitrary deductive systems, indicating which tools of abstract algebraic logic (see Czelakowski [15] and Font, Jansana, and Pigozzi [21]) are really needed at various stages of the theory, while also supplying some new results. The paper is largely self-contained, but its purpose is not to survey the

Received May 1, 2012; accepted June 20, 2013

First published online September 1, 2016

2010 Mathematics Subject Classification: Primary 03B22, 03G27; Secondary 03B47, 08C10

Keywords: deductive system, admissible rule, reduced matrix, structural completeness, Leibniz hierarchy, [order] algebraizable logic, BCIW

(C) 2016 by University of Notre Dame 10.1215/00294527-3671151 


\title{
Admissible Rules and the Leibniz Hierarchy
}

\author{
James G. Raftery
}

\begin{abstract}
This paper provides a semantic analysis of admissible rules and associated completeness conditions for arbitrary deductive systems, using the framework of abstract algebraic logic. Algebraizability is not assumed, so the meaning and significance of the principal notions vary with the level of the Leibniz hierarchy at which they are presented. As a case study of the resulting theory, the nonalgebraizable fragments of relevance logic are considered.
\end{abstract}

\section{Introduction}

Many researchers have considered the question: to what extent can we interpret a logic plausibly in its own metalanguage? Disjunction properties are one manifestation of this concern. A problem in the reverse spirit is the derivability of admissible rules. Following Lorenzen [29], we say that a rule of inference is admissible in a formal system if its addition to the system produces no new theorems. A simple example is the rule of necessitation, $x / \square x$, which is admissible (and not derivable) in quasinormal modal logics. Less trivially, the process of cut-elimination shows that underivable cut rules are admissible in suitable sequent calculi.

The algebraizable logics of Blok and Pigozzi [7] constitute the framework for some prominent treatments of admissibility, such as Rybakov's monograph [62]. On the other hand, the quasinormal modal systems and the cut-free subsystems of substructural logics are not algebraizable. The present paper analyzes the semantics of admissible rules in the context of arbitrary deductive systems, indicating which tools of abstract algebraic logic (see Czelakowski [15] and Font, Jansana, and Pigozzi [21]) are really needed at various stages of the theory, while also supplying some new results. The paper is largely self-contained, but its purpose is not to survey the

Received May 1, 2012; accepted June 20, 2013

First published online September 1, 2016

2010 Mathematics Subject Classification: Primary 03B22, 03G27; Secondary 03B47, 08C10

Keywords: deductive system, admissible rule, reduced matrix, structural completeness, Leibniz hierarchy, [order] algebraizable logic, BCIW

(C) 2016 by University of Notre Dame 10.1215/00294527-3671151 


\title{
Admissible Rules and the Leibniz Hierarchy
}

\author{
James G. Raftery
}

\begin{abstract}
This paper provides a semantic analysis of admissible rules and associated completeness conditions for arbitrary deductive systems, using the framework of abstract algebraic logic. Algebraizability is not assumed, so the meaning and significance of the principal notions vary with the level of the Leibniz hierarchy at which they are presented. As a case study of the resulting theory, the nonalgebraizable fragments of relevance logic are considered.
\end{abstract}

\section{Introduction}

Many researchers have considered the question: to what extent can we interpret a logic plausibly in its own metalanguage? Disjunction properties are one manifestation of this concern. A problem in the reverse spirit is the derivability of admissible rules. Following Lorenzen [29], we say that a rule of inference is admissible in a formal system if its addition to the system produces no new theorems. A simple example is the rule of necessitation, $x / \square x$, which is admissible (and not derivable) in quasinormal modal logics. Less trivially, the process of cut-elimination shows that underivable cut rules are admissible in suitable sequent calculi.

The algebraizable logics of Blok and Pigozzi [7] constitute the framework for some prominent treatments of admissibility, such as Rybakov's monograph [62]. On the other hand, the quasinormal modal systems and the cut-free subsystems of substructural logics are not algebraizable. The present paper analyzes the semantics of admissible rules in the context of arbitrary deductive systems, indicating which tools of abstract algebraic logic (see Czelakowski [15] and Font, Jansana, and Pigozzi [21]) are really needed at various stages of the theory, while also supplying some new results. The paper is largely self-contained, but its purpose is not to survey the

Received May 1, 2012; accepted June 20, 2013

First published online September 1, 2016

2010 Mathematics Subject Classification: Primary 03B22, 03G27; Secondary 03B47, 08C10

Keywords: deductive system, admissible rule, reduced matrix, structural completeness, Leibniz hierarchy, [order] algebraizable logic, BCIW

(C) 2016 by University of Notre Dame 10.1215/00294527-3671151 


\title{
Admissible Rules and the Leibniz Hierarchy
}

\author{
James G. Raftery
}

\begin{abstract}
This paper provides a semantic analysis of admissible rules and associated completeness conditions for arbitrary deductive systems, using the framework of abstract algebraic logic. Algebraizability is not assumed, so the meaning and significance of the principal notions vary with the level of the Leibniz hierarchy at which they are presented. As a case study of the resulting theory, the nonalgebraizable fragments of relevance logic are considered.
\end{abstract}

\section{Introduction}

Many researchers have considered the question: to what extent can we interpret a logic plausibly in its own metalanguage? Disjunction properties are one manifestation of this concern. A problem in the reverse spirit is the derivability of admissible rules. Following Lorenzen [29], we say that a rule of inference is admissible in a formal system if its addition to the system produces no new theorems. A simple example is the rule of necessitation, $x / \square x$, which is admissible (and not derivable) in quasinormal modal logics. Less trivially, the process of cut-elimination shows that underivable cut rules are admissible in suitable sequent calculi.

The algebraizable logics of Blok and Pigozzi [7] constitute the framework for some prominent treatments of admissibility, such as Rybakov's monograph [62]. On the other hand, the quasinormal modal systems and the cut-free subsystems of substructural logics are not algebraizable. The present paper analyzes the semantics of admissible rules in the context of arbitrary deductive systems, indicating which tools of abstract algebraic logic (see Czelakowski [15] and Font, Jansana, and Pigozzi [21]) are really needed at various stages of the theory, while also supplying some new results. The paper is largely self-contained, but its purpose is not to survey the

Received May 1, 2012; accepted June 20, 2013

First published online September 1, 2016

2010 Mathematics Subject Classification: Primary 03B22, 03G27; Secondary 03B47, 08C10

Keywords: deductive system, admissible rule, reduced matrix, structural completeness, Leibniz hierarchy, [order] algebraizable logic, BCIW

(C) 2016 by University of Notre Dame 10.1215/00294527-3671151 


\title{
Admissible Rules and the Leibniz Hierarchy
}

\author{
James G. Raftery
}

\begin{abstract}
This paper provides a semantic analysis of admissible rules and associated completeness conditions for arbitrary deductive systems, using the framework of abstract algebraic logic. Algebraizability is not assumed, so the meaning and significance of the principal notions vary with the level of the Leibniz hierarchy at which they are presented. As a case study of the resulting theory, the nonalgebraizable fragments of relevance logic are considered.
\end{abstract}

\section{Introduction}

Many researchers have considered the question: to what extent can we interpret a logic plausibly in its own metalanguage? Disjunction properties are one manifestation of this concern. A problem in the reverse spirit is the derivability of admissible rules. Following Lorenzen [29], we say that a rule of inference is admissible in a formal system if its addition to the system produces no new theorems. A simple example is the rule of necessitation, $x / \square x$, which is admissible (and not derivable) in quasinormal modal logics. Less trivially, the process of cut-elimination shows that underivable cut rules are admissible in suitable sequent calculi.

The algebraizable logics of Blok and Pigozzi [7] constitute the framework for some prominent treatments of admissibility, such as Rybakov's monograph [62]. On the other hand, the quasinormal modal systems and the cut-free subsystems of substructural logics are not algebraizable. The present paper analyzes the semantics of admissible rules in the context of arbitrary deductive systems, indicating which tools of abstract algebraic logic (see Czelakowski [15] and Font, Jansana, and Pigozzi [21]) are really needed at various stages of the theory, while also supplying some new results. The paper is largely self-contained, but its purpose is not to survey the

Received May 1, 2012; accepted June 20, 2013

First published online September 1, 2016

2010 Mathematics Subject Classification: Primary 03B22, 03G27; Secondary 03B47, 08C10

Keywords: deductive system, admissible rule, reduced matrix, structural completeness, Leibniz hierarchy, [order] algebraizable logic, BCIW

(C) 2016 by University of Notre Dame 10.1215/00294527-3671151 


\title{
Admissible Rules and the Leibniz Hierarchy
}

\author{
James G. Raftery
}

\begin{abstract}
This paper provides a semantic analysis of admissible rules and associated completeness conditions for arbitrary deductive systems, using the framework of abstract algebraic logic. Algebraizability is not assumed, so the meaning and significance of the principal notions vary with the level of the Leibniz hierarchy at which they are presented. As a case study of the resulting theory, the nonalgebraizable fragments of relevance logic are considered.
\end{abstract}

\section{Introduction}

Many researchers have considered the question: to what extent can we interpret a logic plausibly in its own metalanguage? Disjunction properties are one manifestation of this concern. A problem in the reverse spirit is the derivability of admissible rules. Following Lorenzen [29], we say that a rule of inference is admissible in a formal system if its addition to the system produces no new theorems. A simple example is the rule of necessitation, $x / \square x$, which is admissible (and not derivable) in quasinormal modal logics. Less trivially, the process of cut-elimination shows that underivable cut rules are admissible in suitable sequent calculi.

The algebraizable logics of Blok and Pigozzi [7] constitute the framework for some prominent treatments of admissibility, such as Rybakov's monograph [62]. On the other hand, the quasinormal modal systems and the cut-free subsystems of substructural logics are not algebraizable. The present paper analyzes the semantics of admissible rules in the context of arbitrary deductive systems, indicating which tools of abstract algebraic logic (see Czelakowski [15] and Font, Jansana, and Pigozzi [21]) are really needed at various stages of the theory, while also supplying some new results. The paper is largely self-contained, but its purpose is not to survey the

Received May 1, 2012; accepted June 20, 2013

First published online September 1, 2016

2010 Mathematics Subject Classification: Primary 03B22, 03G27; Secondary 03B47, 08C10

Keywords: deductive system, admissible rule, reduced matrix, structural completeness, Leibniz hierarchy, [order] algebraizable logic, BCIW

(C) 2016 by University of Notre Dame 10.1215/00294527-3671151 


\title{
Admissible Rules and the Leibniz Hierarchy
}

\author{
James G. Raftery
}

\begin{abstract}
This paper provides a semantic analysis of admissible rules and associated completeness conditions for arbitrary deductive systems, using the framework of abstract algebraic logic. Algebraizability is not assumed, so the meaning and significance of the principal notions vary with the level of the Leibniz hierarchy at which they are presented. As a case study of the resulting theory, the nonalgebraizable fragments of relevance logic are considered.
\end{abstract}

\section{Introduction}

Many researchers have considered the question: to what extent can we interpret a logic plausibly in its own metalanguage? Disjunction properties are one manifestation of this concern. A problem in the reverse spirit is the derivability of admissible rules. Following Lorenzen [29], we say that a rule of inference is admissible in a formal system if its addition to the system produces no new theorems. A simple example is the rule of necessitation, $x / \square x$, which is admissible (and not derivable) in quasinormal modal logics. Less trivially, the process of cut-elimination shows that underivable cut rules are admissible in suitable sequent calculi.

The algebraizable logics of Blok and Pigozzi [7] constitute the framework for some prominent treatments of admissibility, such as Rybakov's monograph [62]. On the other hand, the quasinormal modal systems and the cut-free subsystems of substructural logics are not algebraizable. The present paper analyzes the semantics of admissible rules in the context of arbitrary deductive systems, indicating which tools of abstract algebraic logic (see Czelakowski [15] and Font, Jansana, and Pigozzi [21]) are really needed at various stages of the theory, while also supplying some new results. The paper is largely self-contained, but its purpose is not to survey the

Received May 1, 2012; accepted June 20, 2013

First published online September 1, 2016

2010 Mathematics Subject Classification: Primary 03B22, 03G27; Secondary 03B47, 08C10

Keywords: deductive system, admissible rule, reduced matrix, structural completeness, Leibniz hierarchy, [order] algebraizable logic, BCIW

(C) 2016 by University of Notre Dame 10.1215/00294527-3671151 


\title{
Admissible Rules and the Leibniz Hierarchy
}

\author{
James G. Raftery
}

\begin{abstract}
This paper provides a semantic analysis of admissible rules and associated completeness conditions for arbitrary deductive systems, using the framework of abstract algebraic logic. Algebraizability is not assumed, so the meaning and significance of the principal notions vary with the level of the Leibniz hierarchy at which they are presented. As a case study of the resulting theory, the nonalgebraizable fragments of relevance logic are considered.
\end{abstract}

\section{Introduction}

Many researchers have considered the question: to what extent can we interpret a logic plausibly in its own metalanguage? Disjunction properties are one manifestation of this concern. A problem in the reverse spirit is the derivability of admissible rules. Following Lorenzen [29], we say that a rule of inference is admissible in a formal system if its addition to the system produces no new theorems. A simple example is the rule of necessitation, $x / \square x$, which is admissible (and not derivable) in quasinormal modal logics. Less trivially, the process of cut-elimination shows that underivable cut rules are admissible in suitable sequent calculi.

The algebraizable logics of Blok and Pigozzi [7] constitute the framework for some prominent treatments of admissibility, such as Rybakov's monograph [62]. On the other hand, the quasinormal modal systems and the cut-free subsystems of substructural logics are not algebraizable. The present paper analyzes the semantics of admissible rules in the context of arbitrary deductive systems, indicating which tools of abstract algebraic logic (see Czelakowski [15] and Font, Jansana, and Pigozzi [21]) are really needed at various stages of the theory, while also supplying some new results. The paper is largely self-contained, but its purpose is not to survey the

Received May 1, 2012; accepted June 20, 2013

First published online September 1, 2016

2010 Mathematics Subject Classification: Primary 03B22, 03G27; Secondary 03B47, 08C10

Keywords: deductive system, admissible rule, reduced matrix, structural completeness, Leibniz hierarchy, [order] algebraizable logic, BCIW

(C) 2016 by University of Notre Dame 10.1215/00294527-3671151 


\title{
Admissible Rules and the Leibniz Hierarchy
}

\author{
James G. Raftery
}

\begin{abstract}
This paper provides a semantic analysis of admissible rules and associated completeness conditions for arbitrary deductive systems, using the framework of abstract algebraic logic. Algebraizability is not assumed, so the meaning and significance of the principal notions vary with the level of the Leibniz hierarchy at which they are presented. As a case study of the resulting theory, the nonalgebraizable fragments of relevance logic are considered.
\end{abstract}

\section{Introduction}

Many researchers have considered the question: to what extent can we interpret a logic plausibly in its own metalanguage? Disjunction properties are one manifestation of this concern. A problem in the reverse spirit is the derivability of admissible rules. Following Lorenzen [29], we say that a rule of inference is admissible in a formal system if its addition to the system produces no new theorems. A simple example is the rule of necessitation, $x / \square x$, which is admissible (and not derivable) in quasinormal modal logics. Less trivially, the process of cut-elimination shows that underivable cut rules are admissible in suitable sequent calculi.

The algebraizable logics of Blok and Pigozzi [7] constitute the framework for some prominent treatments of admissibility, such as Rybakov's monograph [62]. On the other hand, the quasinormal modal systems and the cut-free subsystems of substructural logics are not algebraizable. The present paper analyzes the semantics of admissible rules in the context of arbitrary deductive systems, indicating which tools of abstract algebraic logic (see Czelakowski [15] and Font, Jansana, and Pigozzi [21]) are really needed at various stages of the theory, while also supplying some new results. The paper is largely self-contained, but its purpose is not to survey the

Received May 1, 2012; accepted June 20, 2013

First published online September 1, 2016

2010 Mathematics Subject Classification: Primary 03B22, 03G27; Secondary 03B47, 08C10

Keywords: deductive system, admissible rule, reduced matrix, structural completeness, Leibniz hierarchy, [order] algebraizable logic, BCIW

(C) 2016 by University of Notre Dame 10.1215/00294527-3671151 


\title{
Admissible Rules and the Leibniz Hierarchy
}

\author{
James G. Raftery
}

\begin{abstract}
This paper provides a semantic analysis of admissible rules and associated completeness conditions for arbitrary deductive systems, using the framework of abstract algebraic logic. Algebraizability is not assumed, so the meaning and significance of the principal notions vary with the level of the Leibniz hierarchy at which they are presented. As a case study of the resulting theory, the nonalgebraizable fragments of relevance logic are considered.
\end{abstract}

\section{Introduction}

Many researchers have considered the question: to what extent can we interpret a logic plausibly in its own metalanguage? Disjunction properties are one manifestation of this concern. A problem in the reverse spirit is the derivability of admissible rules. Following Lorenzen [29], we say that a rule of inference is admissible in a formal system if its addition to the system produces no new theorems. A simple example is the rule of necessitation, $x / \square x$, which is admissible (and not derivable) in quasinormal modal logics. Less trivially, the process of cut-elimination shows that underivable cut rules are admissible in suitable sequent calculi.

The algebraizable logics of Blok and Pigozzi [7] constitute the framework for some prominent treatments of admissibility, such as Rybakov's monograph [62]. On the other hand, the quasinormal modal systems and the cut-free subsystems of substructural logics are not algebraizable. The present paper analyzes the semantics of admissible rules in the context of arbitrary deductive systems, indicating which tools of abstract algebraic logic (see Czelakowski [15] and Font, Jansana, and Pigozzi [21]) are really needed at various stages of the theory, while also supplying some new results. The paper is largely self-contained, but its purpose is not to survey the

Received May 1, 2012; accepted June 20, 2013

First published online September 1, 2016

2010 Mathematics Subject Classification: Primary 03B22, 03G27; Secondary 03B47, 08C10

Keywords: deductive system, admissible rule, reduced matrix, structural completeness, Leibniz hierarchy, [order] algebraizable logic, BCIW

(C) 2016 by University of Notre Dame 10.1215/00294527-3671151 


\title{
Admissible Rules and the Leibniz Hierarchy
}

\author{
James G. Raftery
}

\begin{abstract}
This paper provides a semantic analysis of admissible rules and associated completeness conditions for arbitrary deductive systems, using the framework of abstract algebraic logic. Algebraizability is not assumed, so the meaning and significance of the principal notions vary with the level of the Leibniz hierarchy at which they are presented. As a case study of the resulting theory, the nonalgebraizable fragments of relevance logic are considered.
\end{abstract}

\section{Introduction}

Many researchers have considered the question: to what extent can we interpret a logic plausibly in its own metalanguage? Disjunction properties are one manifestation of this concern. A problem in the reverse spirit is the derivability of admissible rules. Following Lorenzen [29], we say that a rule of inference is admissible in a formal system if its addition to the system produces no new theorems. A simple example is the rule of necessitation, $x / \square x$, which is admissible (and not derivable) in quasinormal modal logics. Less trivially, the process of cut-elimination shows that underivable cut rules are admissible in suitable sequent calculi.

The algebraizable logics of Blok and Pigozzi [7] constitute the framework for some prominent treatments of admissibility, such as Rybakov's monograph [62]. On the other hand, the quasinormal modal systems and the cut-free subsystems of substructural logics are not algebraizable. The present paper analyzes the semantics of admissible rules in the context of arbitrary deductive systems, indicating which tools of abstract algebraic logic (see Czelakowski [15] and Font, Jansana, and Pigozzi [21]) are really needed at various stages of the theory, while also supplying some new results. The paper is largely self-contained, but its purpose is not to survey the

Received May 1, 2012; accepted June 20, 2013

First published online September 1, 2016

2010 Mathematics Subject Classification: Primary 03B22, 03G27; Secondary 03B47, 08C10

Keywords: deductive system, admissible rule, reduced matrix, structural completeness, Leibniz hierarchy, [order] algebraizable logic, BCIW

(C) 2016 by University of Notre Dame 10.1215/00294527-3671151 


\title{
Admissible Rules and the Leibniz Hierarchy
}

\author{
James G. Raftery
}

\begin{abstract}
This paper provides a semantic analysis of admissible rules and associated completeness conditions for arbitrary deductive systems, using the framework of abstract algebraic logic. Algebraizability is not assumed, so the meaning and significance of the principal notions vary with the level of the Leibniz hierarchy at which they are presented. As a case study of the resulting theory, the nonalgebraizable fragments of relevance logic are considered.
\end{abstract}

\section{Introduction}

Many researchers have considered the question: to what extent can we interpret a logic plausibly in its own metalanguage? Disjunction properties are one manifestation of this concern. A problem in the reverse spirit is the derivability of admissible rules. Following Lorenzen [29], we say that a rule of inference is admissible in a formal system if its addition to the system produces no new theorems. A simple example is the rule of necessitation, $x / \square x$, which is admissible (and not derivable) in quasinormal modal logics. Less trivially, the process of cut-elimination shows that underivable cut rules are admissible in suitable sequent calculi.

The algebraizable logics of Blok and Pigozzi [7] constitute the framework for some prominent treatments of admissibility, such as Rybakov's monograph [62]. On the other hand, the quasinormal modal systems and the cut-free subsystems of substructural logics are not algebraizable. The present paper analyzes the semantics of admissible rules in the context of arbitrary deductive systems, indicating which tools of abstract algebraic logic (see Czelakowski [15] and Font, Jansana, and Pigozzi [21]) are really needed at various stages of the theory, while also supplying some new results. The paper is largely self-contained, but its purpose is not to survey the

Received May 1, 2012; accepted June 20, 2013

First published online September 1, 2016

2010 Mathematics Subject Classification: Primary 03B22, 03G27; Secondary 03B47, 08C10

Keywords: deductive system, admissible rule, reduced matrix, structural completeness, Leibniz hierarchy, [order] algebraizable logic, BCIW

(C) 2016 by University of Notre Dame 10.1215/00294527-3671151 


\title{
Admissible Rules and the Leibniz Hierarchy
}

\author{
James G. Raftery
}

\begin{abstract}
This paper provides a semantic analysis of admissible rules and associated completeness conditions for arbitrary deductive systems, using the framework of abstract algebraic logic. Algebraizability is not assumed, so the meaning and significance of the principal notions vary with the level of the Leibniz hierarchy at which they are presented. As a case study of the resulting theory, the nonalgebraizable fragments of relevance logic are considered.
\end{abstract}

\section{Introduction}

Many researchers have considered the question: to what extent can we interpret a logic plausibly in its own metalanguage? Disjunction properties are one manifestation of this concern. A problem in the reverse spirit is the derivability of admissible rules. Following Lorenzen [29], we say that a rule of inference is admissible in a formal system if its addition to the system produces no new theorems. A simple example is the rule of necessitation, $x / \square x$, which is admissible (and not derivable) in quasinormal modal logics. Less trivially, the process of cut-elimination shows that underivable cut rules are admissible in suitable sequent calculi.

The algebraizable logics of Blok and Pigozzi [7] constitute the framework for some prominent treatments of admissibility, such as Rybakov's monograph [62]. On the other hand, the quasinormal modal systems and the cut-free subsystems of substructural logics are not algebraizable. The present paper analyzes the semantics of admissible rules in the context of arbitrary deductive systems, indicating which tools of abstract algebraic logic (see Czelakowski [15] and Font, Jansana, and Pigozzi [21]) are really needed at various stages of the theory, while also supplying some new results. The paper is largely self-contained, but its purpose is not to survey the

Received May 1, 2012; accepted June 20, 2013

First published online September 1, 2016

2010 Mathematics Subject Classification: Primary 03B22, 03G27; Secondary 03B47, 08C10

Keywords: deductive system, admissible rule, reduced matrix, structural completeness, Leibniz hierarchy, [order] algebraizable logic, BCIW

(C) 2016 by University of Notre Dame 10.1215/00294527-3671151 


\title{
Admissible Rules and the Leibniz Hierarchy
}

\author{
James G. Raftery
}

\begin{abstract}
This paper provides a semantic analysis of admissible rules and associated completeness conditions for arbitrary deductive systems, using the framework of abstract algebraic logic. Algebraizability is not assumed, so the meaning and significance of the principal notions vary with the level of the Leibniz hierarchy at which they are presented. As a case study of the resulting theory, the nonalgebraizable fragments of relevance logic are considered.
\end{abstract}

\section{Introduction}

Many researchers have considered the question: to what extent can we interpret a logic plausibly in its own metalanguage? Disjunction properties are one manifestation of this concern. A problem in the reverse spirit is the derivability of admissible rules. Following Lorenzen [29], we say that a rule of inference is admissible in a formal system if its addition to the system produces no new theorems. A simple example is the rule of necessitation, $x / \square x$, which is admissible (and not derivable) in quasinormal modal logics. Less trivially, the process of cut-elimination shows that underivable cut rules are admissible in suitable sequent calculi.

The algebraizable logics of Blok and Pigozzi [7] constitute the framework for some prominent treatments of admissibility, such as Rybakov's monograph [62]. On the other hand, the quasinormal modal systems and the cut-free subsystems of substructural logics are not algebraizable. The present paper analyzes the semantics of admissible rules in the context of arbitrary deductive systems, indicating which tools of abstract algebraic logic (see Czelakowski [15] and Font, Jansana, and Pigozzi [21]) are really needed at various stages of the theory, while also supplying some new results. The paper is largely self-contained, but its purpose is not to survey the

Received May 1, 2012; accepted June 20, 2013

First published online September 1, 2016

2010 Mathematics Subject Classification: Primary 03B22, 03G27; Secondary 03B47, 08C10

Keywords: deductive system, admissible rule, reduced matrix, structural completeness, Leibniz hierarchy, [order] algebraizable logic, BCIW

(C) 2016 by University of Notre Dame 10.1215/00294527-3671151 


\title{
Admissible Rules and the Leibniz Hierarchy
}

\author{
James G. Raftery
}

\begin{abstract}
This paper provides a semantic analysis of admissible rules and associated completeness conditions for arbitrary deductive systems, using the framework of abstract algebraic logic. Algebraizability is not assumed, so the meaning and significance of the principal notions vary with the level of the Leibniz hierarchy at which they are presented. As a case study of the resulting theory, the nonalgebraizable fragments of relevance logic are considered.
\end{abstract}

\section{Introduction}

Many researchers have considered the question: to what extent can we interpret a logic plausibly in its own metalanguage? Disjunction properties are one manifestation of this concern. A problem in the reverse spirit is the derivability of admissible rules. Following Lorenzen [29], we say that a rule of inference is admissible in a formal system if its addition to the system produces no new theorems. A simple example is the rule of necessitation, $x / \square x$, which is admissible (and not derivable) in quasinormal modal logics. Less trivially, the process of cut-elimination shows that underivable cut rules are admissible in suitable sequent calculi.

The algebraizable logics of Blok and Pigozzi [7] constitute the framework for some prominent treatments of admissibility, such as Rybakov's monograph [62]. On the other hand, the quasinormal modal systems and the cut-free subsystems of substructural logics are not algebraizable. The present paper analyzes the semantics of admissible rules in the context of arbitrary deductive systems, indicating which tools of abstract algebraic logic (see Czelakowski [15] and Font, Jansana, and Pigozzi [21]) are really needed at various stages of the theory, while also supplying some new results. The paper is largely self-contained, but its purpose is not to survey the

Received May 1, 2012; accepted June 20, 2013

First published online September 1, 2016

2010 Mathematics Subject Classification: Primary 03B22, 03G27; Secondary 03B47, 08C10

Keywords: deductive system, admissible rule, reduced matrix, structural completeness, Leibniz hierarchy, [order] algebraizable logic, BCIW

(C) 2016 by University of Notre Dame 10.1215/00294527-3671151 


\title{
Admissible Rules and the Leibniz Hierarchy
}

\author{
James G. Raftery
}

\begin{abstract}
This paper provides a semantic analysis of admissible rules and associated completeness conditions for arbitrary deductive systems, using the framework of abstract algebraic logic. Algebraizability is not assumed, so the meaning and significance of the principal notions vary with the level of the Leibniz hierarchy at which they are presented. As a case study of the resulting theory, the nonalgebraizable fragments of relevance logic are considered.
\end{abstract}

\section{Introduction}

Many researchers have considered the question: to what extent can we interpret a logic plausibly in its own metalanguage? Disjunction properties are one manifestation of this concern. A problem in the reverse spirit is the derivability of admissible rules. Following Lorenzen [29], we say that a rule of inference is admissible in a formal system if its addition to the system produces no new theorems. A simple example is the rule of necessitation, $x / \square x$, which is admissible (and not derivable) in quasinormal modal logics. Less trivially, the process of cut-elimination shows that underivable cut rules are admissible in suitable sequent calculi.

The algebraizable logics of Blok and Pigozzi [7] constitute the framework for some prominent treatments of admissibility, such as Rybakov's monograph [62]. On the other hand, the quasinormal modal systems and the cut-free subsystems of substructural logics are not algebraizable. The present paper analyzes the semantics of admissible rules in the context of arbitrary deductive systems, indicating which tools of abstract algebraic logic (see Czelakowski [15] and Font, Jansana, and Pigozzi [21]) are really needed at various stages of the theory, while also supplying some new results. The paper is largely self-contained, but its purpose is not to survey the

Received May 1, 2012; accepted June 20, 2013

First published online September 1, 2016

2010 Mathematics Subject Classification: Primary 03B22, 03G27; Secondary 03B47, 08C10

Keywords: deductive system, admissible rule, reduced matrix, structural completeness, Leibniz hierarchy, [order] algebraizable logic, BCIW

(C) 2016 by University of Notre Dame 10.1215/00294527-3671151 


\title{
Admissible Rules and the Leibniz Hierarchy
}

\author{
James G. Raftery
}

\begin{abstract}
This paper provides a semantic analysis of admissible rules and associated completeness conditions for arbitrary deductive systems, using the framework of abstract algebraic logic. Algebraizability is not assumed, so the meaning and significance of the principal notions vary with the level of the Leibniz hierarchy at which they are presented. As a case study of the resulting theory, the nonalgebraizable fragments of relevance logic are considered.
\end{abstract}

\section{Introduction}

Many researchers have considered the question: to what extent can we interpret a logic plausibly in its own metalanguage? Disjunction properties are one manifestation of this concern. A problem in the reverse spirit is the derivability of admissible rules. Following Lorenzen [29], we say that a rule of inference is admissible in a formal system if its addition to the system produces no new theorems. A simple example is the rule of necessitation, $x / \square x$, which is admissible (and not derivable) in quasinormal modal logics. Less trivially, the process of cut-elimination shows that underivable cut rules are admissible in suitable sequent calculi.

The algebraizable logics of Blok and Pigozzi [7] constitute the framework for some prominent treatments of admissibility, such as Rybakov's monograph [62]. On the other hand, the quasinormal modal systems and the cut-free subsystems of substructural logics are not algebraizable. The present paper analyzes the semantics of admissible rules in the context of arbitrary deductive systems, indicating which tools of abstract algebraic logic (see Czelakowski [15] and Font, Jansana, and Pigozzi [21]) are really needed at various stages of the theory, while also supplying some new results. The paper is largely self-contained, but its purpose is not to survey the

Received May 1, 2012; accepted June 20, 2013

First published online September 1, 2016

2010 Mathematics Subject Classification: Primary 03B22, 03G27; Secondary 03B47, 08C10

Keywords: deductive system, admissible rule, reduced matrix, structural completeness, Leibniz hierarchy, [order] algebraizable logic, BCIW

(C) 2016 by University of Notre Dame 10.1215/00294527-3671151 


\title{
Admissible Rules and the Leibniz Hierarchy
}

\author{
James G. Raftery
}

\begin{abstract}
This paper provides a semantic analysis of admissible rules and associated completeness conditions for arbitrary deductive systems, using the framework of abstract algebraic logic. Algebraizability is not assumed, so the meaning and significance of the principal notions vary with the level of the Leibniz hierarchy at which they are presented. As a case study of the resulting theory, the nonalgebraizable fragments of relevance logic are considered.
\end{abstract}

\section{Introduction}

Many researchers have considered the question: to what extent can we interpret a logic plausibly in its own metalanguage? Disjunction properties are one manifestation of this concern. A problem in the reverse spirit is the derivability of admissible rules. Following Lorenzen [29], we say that a rule of inference is admissible in a formal system if its addition to the system produces no new theorems. A simple example is the rule of necessitation, $x / \square x$, which is admissible (and not derivable) in quasinormal modal logics. Less trivially, the process of cut-elimination shows that underivable cut rules are admissible in suitable sequent calculi.

The algebraizable logics of Blok and Pigozzi [7] constitute the framework for some prominent treatments of admissibility, such as Rybakov's monograph [62]. On the other hand, the quasinormal modal systems and the cut-free subsystems of substructural logics are not algebraizable. The present paper analyzes the semantics of admissible rules in the context of arbitrary deductive systems, indicating which tools of abstract algebraic logic (see Czelakowski [15] and Font, Jansana, and Pigozzi [21]) are really needed at various stages of the theory, while also supplying some new results. The paper is largely self-contained, but its purpose is not to survey the

Received May 1, 2012; accepted June 20, 2013

First published online September 1, 2016

2010 Mathematics Subject Classification: Primary 03B22, 03G27; Secondary 03B47, 08C10

Keywords: deductive system, admissible rule, reduced matrix, structural completeness, Leibniz hierarchy, [order] algebraizable logic, BCIW

(C) 2016 by University of Notre Dame 10.1215/00294527-3671151 


\title{
Admissible Rules and the Leibniz Hierarchy
}

\author{
James G. Raftery
}

\begin{abstract}
This paper provides a semantic analysis of admissible rules and associated completeness conditions for arbitrary deductive systems, using the framework of abstract algebraic logic. Algebraizability is not assumed, so the meaning and significance of the principal notions vary with the level of the Leibniz hierarchy at which they are presented. As a case study of the resulting theory, the nonalgebraizable fragments of relevance logic are considered.
\end{abstract}

\section{Introduction}

Many researchers have considered the question: to what extent can we interpret a logic plausibly in its own metalanguage? Disjunction properties are one manifestation of this concern. A problem in the reverse spirit is the derivability of admissible rules. Following Lorenzen [29], we say that a rule of inference is admissible in a formal system if its addition to the system produces no new theorems. A simple example is the rule of necessitation, $x / \square x$, which is admissible (and not derivable) in quasinormal modal logics. Less trivially, the process of cut-elimination shows that underivable cut rules are admissible in suitable sequent calculi.

The algebraizable logics of Blok and Pigozzi [7] constitute the framework for some prominent treatments of admissibility, such as Rybakov's monograph [62]. On the other hand, the quasinormal modal systems and the cut-free subsystems of substructural logics are not algebraizable. The present paper analyzes the semantics of admissible rules in the context of arbitrary deductive systems, indicating which tools of abstract algebraic logic (see Czelakowski [15] and Font, Jansana, and Pigozzi [21]) are really needed at various stages of the theory, while also supplying some new results. The paper is largely self-contained, but its purpose is not to survey the

Received May 1, 2012; accepted June 20, 2013

First published online September 1, 2016

2010 Mathematics Subject Classification: Primary 03B22, 03G27; Secondary 03B47, 08C10

Keywords: deductive system, admissible rule, reduced matrix, structural completeness, Leibniz hierarchy, [order] algebraizable logic, BCIW

(C) 2016 by University of Notre Dame 10.1215/00294527-3671151 


\title{
Admissible Rules and the Leibniz Hierarchy
}

\author{
James G. Raftery
}

\begin{abstract}
This paper provides a semantic analysis of admissible rules and associated completeness conditions for arbitrary deductive systems, using the framework of abstract algebraic logic. Algebraizability is not assumed, so the meaning and significance of the principal notions vary with the level of the Leibniz hierarchy at which they are presented. As a case study of the resulting theory, the nonalgebraizable fragments of relevance logic are considered.
\end{abstract}

\section{Introduction}

Many researchers have considered the question: to what extent can we interpret a logic plausibly in its own metalanguage? Disjunction properties are one manifestation of this concern. A problem in the reverse spirit is the derivability of admissible rules. Following Lorenzen [29], we say that a rule of inference is admissible in a formal system if its addition to the system produces no new theorems. A simple example is the rule of necessitation, $x / \square x$, which is admissible (and not derivable) in quasinormal modal logics. Less trivially, the process of cut-elimination shows that underivable cut rules are admissible in suitable sequent calculi.

The algebraizable logics of Blok and Pigozzi [7] constitute the framework for some prominent treatments of admissibility, such as Rybakov's monograph [62]. On the other hand, the quasinormal modal systems and the cut-free subsystems of substructural logics are not algebraizable. The present paper analyzes the semantics of admissible rules in the context of arbitrary deductive systems, indicating which tools of abstract algebraic logic (see Czelakowski [15] and Font, Jansana, and Pigozzi [21]) are really needed at various stages of the theory, while also supplying some new results. The paper is largely self-contained, but its purpose is not to survey the

Received May 1, 2012; accepted June 20, 2013

First published online September 1, 2016

2010 Mathematics Subject Classification: Primary 03B22, 03G27; Secondary 03B47, 08C10

Keywords: deductive system, admissible rule, reduced matrix, structural completeness, Leibniz hierarchy, [order] algebraizable logic, BCIW

(C) 2016 by University of Notre Dame 10.1215/00294527-3671151 


\title{
Admissible Rules and the Leibniz Hierarchy
}

\author{
James G. Raftery
}

\begin{abstract}
This paper provides a semantic analysis of admissible rules and associated completeness conditions for arbitrary deductive systems, using the framework of abstract algebraic logic. Algebraizability is not assumed, so the meaning and significance of the principal notions vary with the level of the Leibniz hierarchy at which they are presented. As a case study of the resulting theory, the nonalgebraizable fragments of relevance logic are considered.
\end{abstract}

\section{Introduction}

Many researchers have considered the question: to what extent can we interpret a logic plausibly in its own metalanguage? Disjunction properties are one manifestation of this concern. A problem in the reverse spirit is the derivability of admissible rules. Following Lorenzen [29], we say that a rule of inference is admissible in a formal system if its addition to the system produces no new theorems. A simple example is the rule of necessitation, $x / \square x$, which is admissible (and not derivable) in quasinormal modal logics. Less trivially, the process of cut-elimination shows that underivable cut rules are admissible in suitable sequent calculi.

The algebraizable logics of Blok and Pigozzi [7] constitute the framework for some prominent treatments of admissibility, such as Rybakov's monograph [62]. On the other hand, the quasinormal modal systems and the cut-free subsystems of substructural logics are not algebraizable. The present paper analyzes the semantics of admissible rules in the context of arbitrary deductive systems, indicating which tools of abstract algebraic logic (see Czelakowski [15] and Font, Jansana, and Pigozzi [21]) are really needed at various stages of the theory, while also supplying some new results. The paper is largely self-contained, but its purpose is not to survey the

Received May 1, 2012; accepted June 20, 2013

First published online September 1, 2016

2010 Mathematics Subject Classification: Primary 03B22, 03G27; Secondary 03B47, 08C10

Keywords: deductive system, admissible rule, reduced matrix, structural completeness, Leibniz hierarchy, [order] algebraizable logic, BCIW

(C) 2016 by University of Notre Dame 10.1215/00294527-3671151 


\title{
Admissible Rules and the Leibniz Hierarchy
}

\author{
James G. Raftery
}

\begin{abstract}
This paper provides a semantic analysis of admissible rules and associated completeness conditions for arbitrary deductive systems, using the framework of abstract algebraic logic. Algebraizability is not assumed, so the meaning and significance of the principal notions vary with the level of the Leibniz hierarchy at which they are presented. As a case study of the resulting theory, the nonalgebraizable fragments of relevance logic are considered.
\end{abstract}

\section{Introduction}

Many researchers have considered the question: to what extent can we interpret a logic plausibly in its own metalanguage? Disjunction properties are one manifestation of this concern. A problem in the reverse spirit is the derivability of admissible rules. Following Lorenzen [29], we say that a rule of inference is admissible in a formal system if its addition to the system produces no new theorems. A simple example is the rule of necessitation, $x / \square x$, which is admissible (and not derivable) in quasinormal modal logics. Less trivially, the process of cut-elimination shows that underivable cut rules are admissible in suitable sequent calculi.

The algebraizable logics of Blok and Pigozzi [7] constitute the framework for some prominent treatments of admissibility, such as Rybakov's monograph [62]. On the other hand, the quasinormal modal systems and the cut-free subsystems of substructural logics are not algebraizable. The present paper analyzes the semantics of admissible rules in the context of arbitrary deductive systems, indicating which tools of abstract algebraic logic (see Czelakowski [15] and Font, Jansana, and Pigozzi [21]) are really needed at various stages of the theory, while also supplying some new results. The paper is largely self-contained, but its purpose is not to survey the

Received May 1, 2012; accepted June 20, 2013

First published online September 1, 2016

2010 Mathematics Subject Classification: Primary 03B22, 03G27; Secondary 03B47, 08C10

Keywords: deductive system, admissible rule, reduced matrix, structural completeness, Leibniz hierarchy, [order] algebraizable logic, BCIW

(C) 2016 by University of Notre Dame 10.1215/00294527-3671151 


\title{
Admissible Rules and the Leibniz Hierarchy
}

\author{
James G. Raftery
}

\begin{abstract}
This paper provides a semantic analysis of admissible rules and associated completeness conditions for arbitrary deductive systems, using the framework of abstract algebraic logic. Algebraizability is not assumed, so the meaning and significance of the principal notions vary with the level of the Leibniz hierarchy at which they are presented. As a case study of the resulting theory, the nonalgebraizable fragments of relevance logic are considered.
\end{abstract}

\section{Introduction}

Many researchers have considered the question: to what extent can we interpret a logic plausibly in its own metalanguage? Disjunction properties are one manifestation of this concern. A problem in the reverse spirit is the derivability of admissible rules. Following Lorenzen [29], we say that a rule of inference is admissible in a formal system if its addition to the system produces no new theorems. A simple example is the rule of necessitation, $x / \square x$, which is admissible (and not derivable) in quasinormal modal logics. Less trivially, the process of cut-elimination shows that underivable cut rules are admissible in suitable sequent calculi.

The algebraizable logics of Blok and Pigozzi [7] constitute the framework for some prominent treatments of admissibility, such as Rybakov's monograph [62]. On the other hand, the quasinormal modal systems and the cut-free subsystems of substructural logics are not algebraizable. The present paper analyzes the semantics of admissible rules in the context of arbitrary deductive systems, indicating which tools of abstract algebraic logic (see Czelakowski [15] and Font, Jansana, and Pigozzi [21]) are really needed at various stages of the theory, while also supplying some new results. The paper is largely self-contained, but its purpose is not to survey the

Received May 1, 2012; accepted June 20, 2013

First published online September 1, 2016

2010 Mathematics Subject Classification: Primary 03B22, 03G27; Secondary 03B47, 08C10

Keywords: deductive system, admissible rule, reduced matrix, structural completeness, Leibniz hierarchy, [order] algebraizable logic, BCIW

(C) 2016 by University of Notre Dame 10.1215/00294527-3671151 


\title{
Admissible Rules and the Leibniz Hierarchy
}

\author{
James G. Raftery
}

\begin{abstract}
This paper provides a semantic analysis of admissible rules and associated completeness conditions for arbitrary deductive systems, using the framework of abstract algebraic logic. Algebraizability is not assumed, so the meaning and significance of the principal notions vary with the level of the Leibniz hierarchy at which they are presented. As a case study of the resulting theory, the nonalgebraizable fragments of relevance logic are considered.
\end{abstract}

\section{Introduction}

Many researchers have considered the question: to what extent can we interpret a logic plausibly in its own metalanguage? Disjunction properties are one manifestation of this concern. A problem in the reverse spirit is the derivability of admissible rules. Following Lorenzen [29], we say that a rule of inference is admissible in a formal system if its addition to the system produces no new theorems. A simple example is the rule of necessitation, $x / \square x$, which is admissible (and not derivable) in quasinormal modal logics. Less trivially, the process of cut-elimination shows that underivable cut rules are admissible in suitable sequent calculi.

The algebraizable logics of Blok and Pigozzi [7] constitute the framework for some prominent treatments of admissibility, such as Rybakov's monograph [62]. On the other hand, the quasinormal modal systems and the cut-free subsystems of substructural logics are not algebraizable. The present paper analyzes the semantics of admissible rules in the context of arbitrary deductive systems, indicating which tools of abstract algebraic logic (see Czelakowski [15] and Font, Jansana, and Pigozzi [21]) are really needed at various stages of the theory, while also supplying some new results. The paper is largely self-contained, but its purpose is not to survey the

Received May 1, 2012; accepted June 20, 2013

First published online September 1, 2016

2010 Mathematics Subject Classification: Primary 03B22, 03G27; Secondary 03B47, 08C10

Keywords: deductive system, admissible rule, reduced matrix, structural completeness, Leibniz hierarchy, [order] algebraizable logic, BCIW

(C) 2016 by University of Notre Dame 10.1215/00294527-3671151 


\title{
Admissible Rules and the Leibniz Hierarchy
}

\author{
James G. Raftery
}

\begin{abstract}
This paper provides a semantic analysis of admissible rules and associated completeness conditions for arbitrary deductive systems, using the framework of abstract algebraic logic. Algebraizability is not assumed, so the meaning and significance of the principal notions vary with the level of the Leibniz hierarchy at which they are presented. As a case study of the resulting theory, the nonalgebraizable fragments of relevance logic are considered.
\end{abstract}

\section{Introduction}

Many researchers have considered the question: to what extent can we interpret a logic plausibly in its own metalanguage? Disjunction properties are one manifestation of this concern. A problem in the reverse spirit is the derivability of admissible rules. Following Lorenzen [29], we say that a rule of inference is admissible in a formal system if its addition to the system produces no new theorems. A simple example is the rule of necessitation, $x / \square x$, which is admissible (and not derivable) in quasinormal modal logics. Less trivially, the process of cut-elimination shows that underivable cut rules are admissible in suitable sequent calculi.

The algebraizable logics of Blok and Pigozzi [7] constitute the framework for some prominent treatments of admissibility, such as Rybakov's monograph [62]. On the other hand, the quasinormal modal systems and the cut-free subsystems of substructural logics are not algebraizable. The present paper analyzes the semantics of admissible rules in the context of arbitrary deductive systems, indicating which tools of abstract algebraic logic (see Czelakowski [15] and Font, Jansana, and Pigozzi [21]) are really needed at various stages of the theory, while also supplying some new results. The paper is largely self-contained, but its purpose is not to survey the

Received May 1, 2012; accepted June 20, 2013

First published online September 1, 2016

2010 Mathematics Subject Classification: Primary 03B22, 03G27; Secondary 03B47, 08C10

Keywords: deductive system, admissible rule, reduced matrix, structural completeness, Leibniz hierarchy, [order] algebraizable logic, BCIW

(C) 2016 by University of Notre Dame 10.1215/00294527-3671151 


\title{
Admissible Rules and the Leibniz Hierarchy
}

\author{
James G. Raftery
}

\begin{abstract}
This paper provides a semantic analysis of admissible rules and associated completeness conditions for arbitrary deductive systems, using the framework of abstract algebraic logic. Algebraizability is not assumed, so the meaning and significance of the principal notions vary with the level of the Leibniz hierarchy at which they are presented. As a case study of the resulting theory, the nonalgebraizable fragments of relevance logic are considered.
\end{abstract}

\section{Introduction}

Many researchers have considered the question: to what extent can we interpret a logic plausibly in its own metalanguage? Disjunction properties are one manifestation of this concern. A problem in the reverse spirit is the derivability of admissible rules. Following Lorenzen [29], we say that a rule of inference is admissible in a formal system if its addition to the system produces no new theorems. A simple example is the rule of necessitation, $x / \square x$, which is admissible (and not derivable) in quasinormal modal logics. Less trivially, the process of cut-elimination shows that underivable cut rules are admissible in suitable sequent calculi.

The algebraizable logics of Blok and Pigozzi [7] constitute the framework for some prominent treatments of admissibility, such as Rybakov's monograph [62]. On the other hand, the quasinormal modal systems and the cut-free subsystems of substructural logics are not algebraizable. The present paper analyzes the semantics of admissible rules in the context of arbitrary deductive systems, indicating which tools of abstract algebraic logic (see Czelakowski [15] and Font, Jansana, and Pigozzi [21]) are really needed at various stages of the theory, while also supplying some new results. The paper is largely self-contained, but its purpose is not to survey the

Received May 1, 2012; accepted June 20, 2013

First published online September 1, 2016

2010 Mathematics Subject Classification: Primary 03B22, 03G27; Secondary 03B47, 08C10

Keywords: deductive system, admissible rule, reduced matrix, structural completeness, Leibniz hierarchy, [order] algebraizable logic, BCIW

(C) 2016 by University of Notre Dame 10.1215/00294527-3671151 


\title{
Admissible Rules and the Leibniz Hierarchy
}

\author{
James G. Raftery
}

\begin{abstract}
This paper provides a semantic analysis of admissible rules and associated completeness conditions for arbitrary deductive systems, using the framework of abstract algebraic logic. Algebraizability is not assumed, so the meaning and significance of the principal notions vary with the level of the Leibniz hierarchy at which they are presented. As a case study of the resulting theory, the nonalgebraizable fragments of relevance logic are considered.
\end{abstract}

\section{Introduction}

Many researchers have considered the question: to what extent can we interpret a logic plausibly in its own metalanguage? Disjunction properties are one manifestation of this concern. A problem in the reverse spirit is the derivability of admissible rules. Following Lorenzen [29], we say that a rule of inference is admissible in a formal system if its addition to the system produces no new theorems. A simple example is the rule of necessitation, $x / \square x$, which is admissible (and not derivable) in quasinormal modal logics. Less trivially, the process of cut-elimination shows that underivable cut rules are admissible in suitable sequent calculi.

The algebraizable logics of Blok and Pigozzi [7] constitute the framework for some prominent treatments of admissibility, such as Rybakov's monograph [62]. On the other hand, the quasinormal modal systems and the cut-free subsystems of substructural logics are not algebraizable. The present paper analyzes the semantics of admissible rules in the context of arbitrary deductive systems, indicating which tools of abstract algebraic logic (see Czelakowski [15] and Font, Jansana, and Pigozzi [21]) are really needed at various stages of the theory, while also supplying some new results. The paper is largely self-contained, but its purpose is not to survey the

Received May 1, 2012; accepted June 20, 2013

First published online September 1, 2016

2010 Mathematics Subject Classification: Primary 03B22, 03G27; Secondary 03B47, 08C10

Keywords: deductive system, admissible rule, reduced matrix, structural completeness, Leibniz hierarchy, [order] algebraizable logic, BCIW

(C) 2016 by University of Notre Dame 10.1215/00294527-3671151 


\title{
Admissible Rules and the Leibniz Hierarchy
}

\author{
James G. Raftery
}

\begin{abstract}
This paper provides a semantic analysis of admissible rules and associated completeness conditions for arbitrary deductive systems, using the framework of abstract algebraic logic. Algebraizability is not assumed, so the meaning and significance of the principal notions vary with the level of the Leibniz hierarchy at which they are presented. As a case study of the resulting theory, the nonalgebraizable fragments of relevance logic are considered.
\end{abstract}

\section{Introduction}

Many researchers have considered the question: to what extent can we interpret a logic plausibly in its own metalanguage? Disjunction properties are one manifestation of this concern. A problem in the reverse spirit is the derivability of admissible rules. Following Lorenzen [29], we say that a rule of inference is admissible in a formal system if its addition to the system produces no new theorems. A simple example is the rule of necessitation, $x / \square x$, which is admissible (and not derivable) in quasinormal modal logics. Less trivially, the process of cut-elimination shows that underivable cut rules are admissible in suitable sequent calculi.

The algebraizable logics of Blok and Pigozzi [7] constitute the framework for some prominent treatments of admissibility, such as Rybakov's monograph [62]. On the other hand, the quasinormal modal systems and the cut-free subsystems of substructural logics are not algebraizable. The present paper analyzes the semantics of admissible rules in the context of arbitrary deductive systems, indicating which tools of abstract algebraic logic (see Czelakowski [15] and Font, Jansana, and Pigozzi [21]) are really needed at various stages of the theory, while also supplying some new results. The paper is largely self-contained, but its purpose is not to survey the

Received May 1, 2012; accepted June 20, 2013

First published online September 1, 2016

2010 Mathematics Subject Classification: Primary 03B22, 03G27; Secondary 03B47, 08C10

Keywords: deductive system, admissible rule, reduced matrix, structural completeness, Leibniz hierarchy, [order] algebraizable logic, BCIW

(C) 2016 by University of Notre Dame 10.1215/00294527-3671151 


\title{
Admissible Rules and the Leibniz Hierarchy
}

\author{
James G. Raftery
}

\begin{abstract}
This paper provides a semantic analysis of admissible rules and associated completeness conditions for arbitrary deductive systems, using the framework of abstract algebraic logic. Algebraizability is not assumed, so the meaning and significance of the principal notions vary with the level of the Leibniz hierarchy at which they are presented. As a case study of the resulting theory, the nonalgebraizable fragments of relevance logic are considered.
\end{abstract}

\section{Introduction}

Many researchers have considered the question: to what extent can we interpret a logic plausibly in its own metalanguage? Disjunction properties are one manifestation of this concern. A problem in the reverse spirit is the derivability of admissible rules. Following Lorenzen [29], we say that a rule of inference is admissible in a formal system if its addition to the system produces no new theorems. A simple example is the rule of necessitation, $x / \square x$, which is admissible (and not derivable) in quasinormal modal logics. Less trivially, the process of cut-elimination shows that underivable cut rules are admissible in suitable sequent calculi.

The algebraizable logics of Blok and Pigozzi [7] constitute the framework for some prominent treatments of admissibility, such as Rybakov's monograph [62]. On the other hand, the quasinormal modal systems and the cut-free subsystems of substructural logics are not algebraizable. The present paper analyzes the semantics of admissible rules in the context of arbitrary deductive systems, indicating which tools of abstract algebraic logic (see Czelakowski [15] and Font, Jansana, and Pigozzi [21]) are really needed at various stages of the theory, while also supplying some new results. The paper is largely self-contained, but its purpose is not to survey the

Received May 1, 2012; accepted June 20, 2013

First published online September 1, 2016

2010 Mathematics Subject Classification: Primary 03B22, 03G27; Secondary 03B47, 08C10

Keywords: deductive system, admissible rule, reduced matrix, structural completeness, Leibniz hierarchy, [order] algebraizable logic, BCIW

(C) 2016 by University of Notre Dame 10.1215/00294527-3671151 


\title{
Admissible Rules and the Leibniz Hierarchy
}

\author{
James G. Raftery
}

\begin{abstract}
This paper provides a semantic analysis of admissible rules and associated completeness conditions for arbitrary deductive systems, using the framework of abstract algebraic logic. Algebraizability is not assumed, so the meaning and significance of the principal notions vary with the level of the Leibniz hierarchy at which they are presented. As a case study of the resulting theory, the nonalgebraizable fragments of relevance logic are considered.
\end{abstract}

\section{Introduction}

Many researchers have considered the question: to what extent can we interpret a logic plausibly in its own metalanguage? Disjunction properties are one manifestation of this concern. A problem in the reverse spirit is the derivability of admissible rules. Following Lorenzen [29], we say that a rule of inference is admissible in a formal system if its addition to the system produces no new theorems. A simple example is the rule of necessitation, $x / \square x$, which is admissible (and not derivable) in quasinormal modal logics. Less trivially, the process of cut-elimination shows that underivable cut rules are admissible in suitable sequent calculi.

The algebraizable logics of Blok and Pigozzi [7] constitute the framework for some prominent treatments of admissibility, such as Rybakov's monograph [62]. On the other hand, the quasinormal modal systems and the cut-free subsystems of substructural logics are not algebraizable. The present paper analyzes the semantics of admissible rules in the context of arbitrary deductive systems, indicating which tools of abstract algebraic logic (see Czelakowski [15] and Font, Jansana, and Pigozzi [21]) are really needed at various stages of the theory, while also supplying some new results. The paper is largely self-contained, but its purpose is not to survey the

Received May 1, 2012; accepted June 20, 2013

First published online September 1, 2016

2010 Mathematics Subject Classification: Primary 03B22, 03G27; Secondary 03B47, 08C10

Keywords: deductive system, admissible rule, reduced matrix, structural completeness, Leibniz hierarchy, [order] algebraizable logic, BCIW

(C) 2016 by University of Notre Dame 10.1215/00294527-3671151 